\title{
Slab magnetised non-relativistic low-beta electron-positron plasmas: collisionless heating, linear waves and reconnecting instabilities
}

\author{
Alessandro Zocco $\dagger$ \\ Max-Planck-Institut für Plasmaphysik, D-17491, Greifswald, Germany \\ (Received 1 August 2017; revised 6 November 2017; accepted 7 November 2017)
}

The properties of a non-relativistic magnetised low-beta electron-positron plasma in slab geometry are investigated. The two species are taken to be drift kinetic while we retain Larmor radius effects in quasi-neutrality, and inertia in Ohm's law. A linear analysis shows that, for small magnetic perturbations, Alfvénic perturbations travel at the electron Alfvén speed, which is based on the electron mass. We discuss the role of the displacement current when Larmor-scale and Debye-scale effects are both retained. We predict the existence of a kinetic electron Alfvén wave which connects to the K-modes of Mishchenko et al. (J. Plasma Phys., 2017 (submitted)) in the electrostatic limit. It is found that linear drift waves are not supported by the system if the two species have the same temperature. Tearing modes can be driven unstable by equilibrium current density gradients. Also in this case, the characteristic time is based on the electron Alfvén speed. Nonlinear hybrid fluid-kinetic equations are also derived. It is shown that each species is described, to leading order, by the kinetic reduced electron heating model (KREHM) kinetic equation of Zocco \& Schekochihin (Phys. Plasmas, vol. 18, 2011, 102309). The model is extended to retain first-order Larmor radius effects. It supports collisionless dispersive waves, which can greatly impact nonlinear magnetic reconnection. Diamagnetic effects enter the nonlinear equations via the first-order magnetic compressibility. A minimal nonlinear model for two-dimensional low-frequency isothermal pair plasmas is derived.

Key words: plasma dynamics, plasma properties

\section{Introduction}

The creation of a magnetically confined laboratory electron-positron plasma (Pedersen et al. 2012; Saitoh et al. 2014) is becoming a new element of inspiration for some researchers in the field of plasma physics. These systems, while apparently exotic (especially for non-relativistic regimes), can contribute to the understanding of some important properties of physical phenomena in electron-ion plasmas. The role of dispersive waves in fast magnetic reconnection in ordinary plasmas, for instance, has been put into perspective by particle-in-cell simulations (Bessho \& Bhattacharjee 2005; Daughton \& Karimabadi 2007) and fluid theory and simulation

$†$ Email address for correspondence: Alessandro.zocco@ipp.mpg.de 
results of electron-positron plasmas (Chacón, Simakov \& Zocco 2007; Zocco, Chacón \& Simakov 2008, 2009). Similarly, the study of pair plasmas can be relevant in other contexts, such as the energy dynamics of decaying turbulence (Makwana et al. 2015; Guo et al. 2016). There is an increasing number of analytical results discussed in the recent literature, but most of these are valid only linearly. Nonlinear results are rare (Helander 2017), and models that combine computational realisability and physical verisimilitude are desirable. In this article we revisit some aspects of recent linear results, and propose a possible route towards a reduced kinetic nonlinear model for electron-positron plasmas. We present some fluid equations similar to those of Chacón et al. (2008), but in the framework of gyrokinetics for magnetic reconnection (Zocco \& Schekochihin 2011; Loureiro, Schekochihin \& Zocco 2013; Zocco 2015; Zocco et al. 2015; Loureiro et al. 2016). The new aspect here introduced is in the quasi-neutrality equation. The smallest kinetic scale that enters the quasi-neutrality equation is taken to be the Larmor scale, $\rho_{e}=v_{\text {the }} / \Omega_{c}$, which is assumed to be much smaller than the inertial scale $d_{e}=\rho_{e} / \sqrt{\beta}$, where $\beta$ is the ratio of kinetic to plasma pressure, $\Omega_{c}=e B /(m c)$ is the cyclotron frequency, $v_{\text {the }}$ the thermal speed and $B$ the strength of the equilibrium magnetic field. The inclusion of the Debye length is avoided (Helander 2014; Helander \& Connor 2016), as this would require a covariant treatment. However, the possible effects of the displacement current are discussed. Some considerations on linear waves and reconnecting instabilities are given in $\S 2$. In $\S 6$, we propose an adaptation of the kinetic reduced electron heating model (KREHM) equations (Zocco \& Schekochihin 2011) to low-beta pair plasmas. Particular attention is devoted to the isothermal limit. Conclusions are in $\S 7$.

\section{Linear analysis}

In our system, density fluctuations for electrons $\left(e^{-}=-|e|\right)$ and positrons $\left(e^{+}=\right.$ $|e|)$ are calculated by taking the zeroth moment of the perturbed distribution function $\delta f_{e^{\mp}}=-e^{\mp} \varphi F_{0} / T_{0}+h_{e^{\mp}}$. The non-adiabatic part of $\delta f$ satisfies the electromagnetic gyrokinetic equation of Frieman \& Chen (1982),

$$
\begin{aligned}
\frac{\mathrm{d} h_{e^{\mp}}}{\mathrm{d} t}+v_{\|} \hat{\boldsymbol{b}} \cdot \nabla h_{e^{\mp}}= & \frac{e^{\mp} F_{0}}{T_{0}} \mathrm{~J}_{0}\left(k_{\perp} \rho_{e} \hat{v}_{\perp}\right) \frac{\partial}{\partial t}\left(\varphi-\frac{v_{\|}}{c} A_{\|}\right) \\
& -\frac{c}{B_{0}} \boldsymbol{e}_{z} \cdot \nabla\left(\varphi-\frac{v_{\|}}{c} A_{\|}\right) \times \nabla F_{0}+\left(\frac{\mathrm{d} h_{e^{\mp}}}{\mathrm{d} t}\right)_{\text {coll }},
\end{aligned}
$$

where $\mathrm{d} / \mathrm{d} t=\partial_{t}+B_{0}^{-1}\left\{\mathrm{~J}_{0} \varphi,\right\}, \hat{\boldsymbol{b}} \cdot \nabla=\partial_{z}-B_{0}^{-1}\left\{\mathrm{~J}_{0} A_{\|},\right\},\{A, B\}=\partial_{x} A \partial_{y} B-\partial_{y} A \partial_{x} B$, $v_{\|}$is the particle velocity in the $z$-direction, parallel to the guide field of modulus $B_{0}, \hat{v}_{\perp}$ is the particle velocity, perpendicular to it, normalised to the thermal speed $v_{\text {the }}=\sqrt{2 T_{0} / m}$, where $T_{0}$ is the reference temperature of the Maxwellian equilibrium $F_{0}, x$ and $y$ are Cartesian space coordinates orthogonal to $z$, and $\mathrm{J}_{0}=\mathrm{J}_{0}\left(k_{\perp} \rho_{e} \hat{v}_{\perp}\right)$ is the Bessel function. Here $\varphi$ and $A_{\|}$are the electrostatic potential, and the parallel component of the magnetic vector potential, respectively. In gyrokinetics, perturbations can vary across the equilibrium magnetic field on a small scale (of the order of the Larmor radius), but equilibrium quantities vary slowly on a macroscopic length, $L$. Furthermore, perturbed quantities vary slowly along the equilibrium magnetic field $k_{\|} / k_{\perp} \equiv \epsilon_{G K} \sim \rho_{e} / L \ll 1$, and evolve on a time scale which is much longer than the inverse cyclotron frequency, $\Omega^{-1}$. Thus, densities evolve following the familiar continuity equation

$$
\frac{\mathrm{d}}{\mathrm{d} t} \frac{\delta n_{e^{\mp}}}{n_{0}}=-\hat{\boldsymbol{b}} \cdot \nabla u_{\| e^{\mp}}-\mathrm{i} \omega_{* e^{\mp}} \frac{e^{\mp} \varphi}{T_{0}},
$$


with $u_{\|, e^{\mp}}=n_{0}^{-1} \int \mathrm{d}^{3} \boldsymbol{v} \delta f_{e^{+}} v_{\|}$. We are using the local approximation

$$
\frac{c}{B_{0}} \nabla \varphi \cdot \frac{\nabla n_{0}}{n_{0}}=-\mathrm{i} \omega_{* e^{\mp}} \frac{e^{\mp} \varphi}{T_{0}}
$$

for the background density gradient, which introduces effects associated with the diamagnetic frequency $\omega_{* e^{\mp}}=\mp \mathrm{i} v_{\text {the }} /\left(2 L_{n}\right) \rho_{e} \partial_{y}$, where $\nabla n_{0} / n_{0} \approx-L_{n}^{-1},(\partial h / \partial t)_{\text {coll }}$ is the collisional operator and $k_{\perp} \rho_{e} \ll 1$.

Poisson's equation for the electrostatic potential, $\varphi$, is derived from the quasineutrality condition

$$
\int \mathrm{d}^{3} \boldsymbol{v} \delta f_{e^{-}}=\int \mathrm{d}^{3} \boldsymbol{v} \delta f_{e^{+}}
$$

We consider the ansatz $h_{e^{\mp}}=\left(e^{\mp} \varphi / T_{0}+\delta n_{e^{\mp}} / n_{0}+2 v_{\|} u_{\| e^{\mp}} / v_{\text {the }}^{2}\right) F_{0}+g_{e^{\mp}}$, where $\int \mathrm{d}^{3} \boldsymbol{v} g_{e^{\mp}}=\int \mathrm{d}^{3} \boldsymbol{v} v_{\|} g_{e^{\mp}} \equiv 0$. Then

$$
2 \frac{T_{0}}{e}\left(\frac{\delta n_{e^{+}}}{n_{0}}-\frac{\delta n_{e^{-}}}{n_{0}}\right)=-\rho_{e}^{2} \nabla^{2} \varphi,
$$

where we see that

$$
\frac{\delta n_{e^{+}}}{n_{0}}-\frac{\delta n_{e^{-}}}{n_{0}} \sim \beta \frac{e \varphi}{T_{0}} \ll \frac{e \varphi}{T_{0}},
$$

if $k_{\perp} d_{e} \sim 1$, with $d_{e}=c / \omega_{p l}, \omega_{p l}^{2}=4 \pi n_{0} e^{2} /\left(c m_{e}\right), \beta=8 \pi n_{0} T_{0} / B^{2} \ll 1$, and

$$
d_{e} \gg \rho_{e} \gg \lambda_{D}
$$

guarantees balance in (2.5). Equation (2.5) is valid, strictly speaking, only if perpendicular temperature fluctuations, associated with finite Larmor radius effects, are neglected. We consider this regime for the moment, and leave a more rigorous treatment of temperature, density and parallel magnetic fluctuations for the section which is concerned with nonlinear physics. Before proceeding, however, we notice that (2.5) is similar to the quasi-neutrality condition used by Helander (2014) where the Debye length is replaced by the Larmor scale. This is a fundamental requirement to avoid the otherwise awkward condition $d_{e} \sim \lambda_{D}$, which would imply $v_{\text {the }} \sim c$. Following Helander \& Connor (2016), we retain perpendicular magnetic perturbations, but neglect magnetic compressibility, $\delta B_{\|}$.

We then calculate the $v_{\|}$-moment of (2.1), to obtain

$$
\begin{aligned}
\frac{\mathrm{d}}{\mathrm{d} t}\left(A_{\|}+\frac{m c}{e^{\mp}} u_{\| e^{\mp}}\right)= & -c \frac{\partial \varphi}{\partial z}-\frac{T_{0} c}{e^{\mp}} \hat{\boldsymbol{b}} \cdot \nabla\left(\frac{\delta n_{e^{\mp}}}{n_{0}}+\frac{\delta T_{\| e^{\mp}}}{T_{0}}\right) \\
& +\mathrm{i} \omega_{* e^{\mp}}\left(1+\eta_{e}\right) A_{\|}+\frac{m c}{e} v\left(u_{\| e^{-}}-u_{\| e^{+}}\right),
\end{aligned}
$$

where $\eta_{e}=n_{0} \nabla T_{0} /\left(T_{0} \nabla n_{0}\right), v$ a constant collision frequency, $\delta T_{\| e^{\mp}}=n_{0}^{-1} \int \mathrm{d} v_{\|} 2 \hat{v}_{\|}^{2} h_{e^{\mp}}$ and a simple Lenard-Bernstein collisional model operator has been used (Lenard \& Bernstein 1958; Zocco \& Schekochihin 2011). Parallel Ampère's law gives

$$
\frac{e}{m c} d_{e}^{2} \nabla^{2} A_{\|}=u_{\| e^{-}}-u_{\| e^{+}}
$$


where the displacement current has been neglected. Equation (2.2), if we take into account of (2.5), implies that

$$
\omega \sim k_{\|} v_{A, e} \quad \text { and } \quad \frac{v_{\text {the }}}{c} A_{\|} \sim \sqrt{\beta} \varphi,
$$

where $v_{A, e}=B / \sqrt{4 \pi m_{e} n_{0}}$ is the Alfvén speed based on the electron mass. Notice that $v_{A, e}$ is defined using the electron density only. The system is closed with an equation for the parallel temperature fluctuations, derived using a highly collisional fluid closure, $v \gg \omega$, for the flux of energy (Zocco \& Schekochihin 2011; Zocco et al. 2015)

$$
\begin{aligned}
\frac{\mathrm{d}}{\mathrm{d} t} \frac{\delta T_{\| e^{\mp}}}{T_{0}}= & \frac{v_{\mathrm{the}}^{2}}{2 v}(\hat{\boldsymbol{b}} \cdot \nabla)^{2} \frac{\delta T_{\| e^{\mp}}}{T_{0}}-\mathrm{i} \frac{v_{\mathrm{the}}^{2}}{2 v} \hat{\boldsymbol{b}} \cdot \nabla \eta_{e^{\mp}} \omega_{* e^{\mp}} \frac{e^{\mp} A_{\|}}{T_{0}} \\
& -\mathrm{i} \eta_{e^{\mp}} \omega_{* e^{\mp}} \frac{e^{\mp} \varphi}{T_{0}}-2 \hat{\boldsymbol{b}} \cdot \nabla u_{\| e^{\mp}} .
\end{aligned}
$$

This is just a choice that facilitates the forthcoming discussion. More precisely, we are considering the semi-collisional limit (Drake \& Lee 1977)

$$
1 \ll \sqrt{\frac{v}{\omega}} \sim \frac{k_{\|} v_{\text {the }}}{\omega} \ll \frac{v}{\omega},
$$

with $^{1} \omega \sim \omega_{* e}$. Electron thermal conduction effects are negligible for

$$
1 \ll \frac{k_{\|} v_{\text {the }}}{\omega} \ll \sqrt{\frac{v}{\omega}},
$$

then (2.11) becomes

$$
\frac{\mathrm{d}}{\mathrm{d} t} \frac{\delta T_{\| e^{\mp}}}{T_{0}} \approx-\mathrm{i} \omega_{* e^{\mp}} \frac{e^{\mp} \varphi}{T_{0}}-2 \hat{\boldsymbol{b}} \cdot \nabla u_{\| e^{\mp}} .
$$

The system could easily be left completely kinetic, then (2.11) would couple to higher-order moments. However, each of these moments would follow a universal equation when projected on the basis of Hermite polynomials which allows for an efficient treatment of the non-isothermal case $\delta T_{\|} \neq 0$ (Zocco \& Schekochihin 2011; Loureiro et al. 2013; Zocco 2015; Zocco et al. 2015; Schekochihin et al. 2016). In the truly collisionless case the hierarchy of Hermite moments generates a plasma response which was proven to be equivalent to the collisionless response evaluated via Landau contour integration (Zocco 2015). The isothermal approximation instead, $\delta T_{\|} \equiv 0$, would be described by the electron response of the nonlinear model of Schep, Pegoraro \& Kuvshinov (1994). In the context of linear magnetic reconnection, the presence of temperature fluctuations is a technicality that has an impact on the transition from collisional to collisionless regimes, but it is irrelevant when one wants to estimate reconnection rates for very small but finite collisionality. The inclusion of the resonant electron response (i.e. Landau resonance) is not necessary to obtain the correct reconnection rates in the collisionless limit, since the relevant condition to

\footnotetext{
${ }^{1}$ The numerical factor does not coincide with the one evaluated by Braginskii (1965), since we are using a collision operator model.
} 
transition into a collisionless reconnection regime is that the inertial scale exceeds the resistive one, and this can occur even when collisions are finite. ${ }^{2}$ For this reason, we are justified in using (2.11) and yet consider a collisionless limit for linear magnetic reconnection. This is a well-known fact for electron-ion plasmas (Ottaviani \& Porcelli 1995), and electron-positron plasmas do not seem to be an exception. Of all the three regimes discussed (highly collisional, truly collisionless, kinetic non-isothermal with finite collisions), the last is certainly the most realistic one. Nonlinearly, the role of high-order moments that couple to the equation for temperature fluctuations is very important, as was shown by Loureiro et al. (2013) for electron-ion plasmas. The evolution of high-order moments of the distribution function is a consequence of the formation of small-scale structures in velocity space, which gives rise to heating even in the limit of vanishing collisionality (in steady state or sufficiently slow dynamics) (Zocco \& Schekochihin 2011). There is no reason to believe that the same phenomenon cannot occur in pair plasmas.

\section{Small thermal conductivity: waves}

We consider the approximation of small thermal conductivity, therefore

$$
\frac{\mathrm{d}}{\mathrm{d} t} \frac{\delta T_{\| e^{\mp}}}{T_{0}} \approx-\mathrm{i} \omega_{* e^{\mp}} \frac{e^{\mp} \varphi}{T_{0}}-2 \hat{\boldsymbol{b}} \cdot \nabla u_{\| e^{\mp}} .
$$

We use (3.1) in (2.8), we then add the parallel moment equations (2.8) of the two species, to notice that diamagnetic effects cancel exactly. Thus, we obtain

$$
A_{\|}-\frac{k_{\|} c}{\omega} \varphi=\frac{v}{\mathrm{i} \omega}\left(1-\mathrm{i} \frac{\omega}{2 v}-\frac{3}{4} \frac{k_{\|}^{2} v_{\text {the }}^{2}}{\mathrm{i} \omega \nu}\right) k_{\perp}^{2} d_{e}^{2} A_{\|} .
$$

On the other hand, Poisson's equation (2.5), after using the continuity equations and Ampère's law (2.9), becomes

$$
\varphi=\frac{1}{2 \beta_{e}} \frac{k_{\|} v_{\text {the }}}{\omega} \frac{v_{\text {the }}}{c} A_{\|} .
$$

By combining (3.2) and (3.3), we obtain

$$
\omega^{2}=\frac{1}{2} \frac{k_{\|}^{2} v_{A, e}^{2}}{1+\left(1+\mathrm{i} \frac{2 v}{\omega}\right) k_{\perp}^{2} d_{e}^{2} / 2},
$$

where we are taking the limit

$$
k_{\perp}^{2} \rho_{e}^{2} \sim \beta \ll 1
$$

Thus, we find no drift wave, a result also obtained by Helander (2014). In the 'collisionless' regime $(\omega \gg v)$ we find the dispersive waves

$$
\omega^{2}=\frac{1}{2} \frac{k_{\|}^{2} v_{A, e}^{2}}{1+k_{\perp}^{2} d_{e}^{2} / 2},
$$

\footnotetext{
${ }^{2}$ See, for instance, Zocco et al. (2015) where the truly collisionless electron conductivity, evaluated via Landau contour integration, is reproduced very well by a truncated continued fraction solution generated by a Hermite expansion of the electron distribution function.
} 
which, at long wavelengths, becomes a shear Alfvén wave,

$$
\omega^{2} \approx \frac{k_{\|}^{2} v_{A, e}^{2}}{2},
$$

where the factor of 2 in the denominator stems from the unconventional definition of $v_{A}$ after (2.10), which only involved half the density.

In the presence of collisions, electron thermal conduction induces a damping at short wavelengths

$$
\omega \approx \frac{-\mathrm{i}}{2 k_{\perp}^{2} d_{e}^{2}} \frac{k_{\|}^{2} v_{A, e}^{2}}{v} .
$$

Perhaps not surprisingly, (3.8) defines the semi-collisional scale introduced by Drake \& Lee (1977).

Had we retained the Debye length instead of the Larmor radius in (2.5) $\left(\rho_{e}^{2} \rightarrow \lambda_{D}^{2}\right)$, we would have found two waves travelling at the speed of light, which we prefer not to allow for, because the displacement current has been neglected in Ampère's law (2.9). This would have been true also in the isothermal limit $\left(\delta T_{\|}=0\right)$. Then, (3.4) would have been

$$
\omega= \pm \frac{k_{\|} c}{\sqrt{2}},
$$

which, again, cannot be accepted. Had one retained the whole hierarchy of moments coupled to (2.11), valid for arbitrary collisionality, they would still have entered the dispersion relation via the $k_{\perp}^{2} \lambda_{D}^{2}$ term and yielded a wave travelling at the speed of light. We conclude that the collisionless electromagnetic limit must be at least Lorentz-Poincaré invariant. As already anticipated, the reason is more apparent if one ponders the consequences of allowing the electrostatic potential to vary on the Debye scale, while letting the current vary on the inertial scale, $d_{e}$. This implies

$$
\lambda_{D} \sim d_{e} \rightarrow v_{\text {the }} \sim c,
$$

which demands a relativistic description. An electromagnetic gyrokinetic theory that retains Larmor radius effects seems to suffer from a similar problem, since in this case

$$
\lambda_{D} \sim \rho_{e} \rightarrow v_{A, e} \sim c,
$$

where $\rho_{e}$ is the Larmor radius and $v_{A, e}$ the Alfvén speed. However, while (3.10) is a condition on the kinetic energy of particles, which can be met in extreme conditions, (3.11) is simply stating that Alfvén waves must be allowed to travel at the speed of light. This is a perfectly acceptable physical requirement provided Maxwell's equations are kept consistent with a covariant description, therefore including the displacement current in Ampère's law. We must stress that all frequencies must then be taken to be much smaller than the cyclotron frequency, as required by a gyrokinetic theory. Some of these aspects have also been pointed out by a recent work of Stenson et al. (2017).

\section{Alfvén waves}

We could insist on keeping a finite Debye length in our electromagnetic equations. In this case, the displacement current in Ampère's law should be retained, since it plays a crucial role in establishing charge neutrality and generating Langmuir waves. In this case our model equations will indeed be suitable for a covariant formulation. 
Thus, we consider a modified version of (2.3b) of Helander \& Connor (2016) where the displacement current has been added

$$
\left(\nabla^{2}-\frac{1}{c^{2}} \frac{\partial^{2}}{\partial t^{2}}\right) A_{\|}-\frac{1}{c} \frac{\partial}{\partial t} \nabla_{\|} \varphi=\frac{4 \pi n_{0} e}{c}\left(u_{\| e^{-}}-u_{\| e^{+}}\right)
$$

This replaces (2.9). Poisson's law, since we are using the radiation (Coulomb) gauge, $\boldsymbol{\nabla} \cdot \boldsymbol{A}=0$, does not change. Let us first show that the collisionless isothermal response derived using our (2.2) and (2.8) gives the same result as (3.7) of Helander \& Connor (2016) for $d_{e}^{-1} \ll k_{\perp} \ll \lambda_{D}^{-1}$, which is the regime of interest of the future APEX experiment (Pedersen et al. 2012; Saitoh et al. 2014). Now quasi-neutrality (2.5) (with $\rho_{e} \rightarrow \sqrt{2} \lambda_{D}$ ) and Ohm's law (2.8) (with $v \rightarrow 0$ ) become

$$
\frac{1}{4} \frac{k_{\|} c}{\omega}\left[\left(k_{\perp}^{2}-\frac{\omega^{2}}{c^{2}}\right) A_{\|}+\frac{\omega}{c} k_{\|} \varphi\right]=k_{\perp}^{2} \varphi
$$

and

$$
\frac{\omega}{k_{\|} c}\left\{A_{\|}+\frac{d_{e}^{2}}{2}\left[\left(k_{\perp}^{2}-\frac{\omega^{2}}{c^{2}}\right) A_{\|}+\frac{\omega}{c} k_{\|} \varphi\right]\right\}=\left(1+k_{\perp}^{2} \lambda_{D}^{2}\right) \varphi .
$$

For $\lambda_{D}^{-1} \gg k_{\perp} \gg d_{e}^{-1}$, when $\lambda_{D} \ll d_{e}$, we obtain a dispersive Langmuir wave

$$
\omega^{2} \approx \frac{k_{\|}^{2}}{2 k_{\perp}^{2}} \omega_{p l}^{2}
$$

where $\omega_{p l}$ is the plasma frequency. Equation (4.4) indeed coincides with the high-frequency limit of the electrostatic wave that solves (3.7) of Helander \& Connor (2016). For the range of wavelengths of interest, the displacement current does not seem to have an effect. A full kinetic treatment would damp this wave (See Mishchenko et al. 2017).

We now turn our attention to Alfvénic perturbations, which should connect to the wave just found in the electrostatic limit, $\lambda_{D} \gg \rho_{e}$. We rewrite Poisson's law and Ampère's law for the collisionless drift-kinetic case ((3.3) and (3.4) of Helander \& Connor 2016)

$$
\left(\begin{array}{ll}
a_{11} & a_{12} \\
a_{21} & a_{22}
\end{array}\right)\left(\begin{array}{c}
\varphi \\
\frac{v_{\text {the }}}{c} A_{\|}
\end{array}\right)=0
$$

where the coefficients were evaluated by Helander \& Connor (2016): $a_{11}=1+\lambda_{D}^{2} k_{\perp}^{2}+$ $\zeta Z(\zeta), a_{22}=-\zeta^{2}[1+\zeta Z(\zeta)]+k_{\perp}^{2} d_{e}^{2}, a_{21}=-a_{12}=\zeta[1+\zeta Z(\zeta)]$, with $\zeta=\omega /\left(k_{\|} v_{\text {the }}\right)$ and $Z$ is the plasma dispersion function (Fried, Hendrick \& McCune 1968). When (4.1) is used instead of (2.9), we have that

$$
k_{\perp}^{2} d_{e}^{2} \rightarrow k_{\perp}^{2} d_{e}^{2}\left(k_{\perp}^{2}-\frac{\omega^{2}}{c^{2}}\right),
$$

and

$$
a_{21} \rightarrow \zeta[1+\zeta Z(\zeta)]+\zeta k_{\|}^{2} \lambda_{D} d_{e}
$$


For Alfvénic perturbations we must expand the plasma dispersion function for large arguments, ${ }^{3}$ since

$$
\zeta=\frac{\omega}{k_{\|} v_{\text {the }}}=\frac{\omega}{\sqrt{\beta} k_{\|} v_{A, e}} \sim \frac{1}{\sqrt{\beta}} \gg 1 .
$$

Setting to zero the determinant of the matrix of (4.5), for $k_{\perp} \lambda_{D} \sim k_{\perp} \rho_{e} \sim \sqrt{\beta} \ll 1$, then gives

$$
\frac{\omega^{2}}{k_{\|}^{2} v_{A, e}^{2}}=\beta \frac{d_{e}^{2}\left(k_{\perp}^{2}-\frac{\omega^{2}}{c^{2}}\right)}{\left(k_{\perp}^{2} \rho_{e}^{2}+k_{\perp}^{2} \lambda_{D}^{2}\right)\left[1+2 d_{e}^{2}\left(k_{\perp}^{2}-\frac{\omega^{2}}{c^{2}}\right)\right]+k_{\|}^{2} \lambda_{D} d_{e}}
$$

which is a kinetically modified Alfvén wave. Since

$$
\frac{\omega}{k_{\perp} c}=\frac{\omega}{k_{\|} v_{A, e}} \frac{v_{A, e}}{c} \epsilon_{G K} \ll 1,
$$

we have

$$
\frac{\omega^{2}}{k_{\|}^{2} v_{A, e}^{2}}=\beta \frac{d_{e}^{2} k_{\perp}^{2}}{\left(k_{\perp}^{2} \rho_{e}^{2}+k_{\perp}^{2} \lambda_{D}^{2}\right)\left[1+2 d_{e}^{2} k_{\perp}^{2}\right]+k_{\|}^{2} \lambda_{D} d_{e}} .
$$

When $\left(k_{\|}^{2} / k_{\perp}^{2}\right) v_{A, e} / c \equiv \epsilon_{G K}^{2} v_{A, e} / c \gg \sqrt{\beta}$, for $\rho_{e} \sim \lambda_{D}$, the $k_{\|}^{2} \lambda_{D} d_{e}$ in the denominator is dominant, and we have

$$
\omega^{2} \approx k_{\perp}^{2} \rho_{e}^{2} \frac{v_{A, e}^{2}}{\lambda_{D} d_{e}} \rightarrow \frac{\omega^{2}}{\Omega_{c}^{2}} \sim \beta^{1 / 4}
$$

However, (4.12) was derived for $\beta^{1 / 4} \ll \epsilon_{G K}$. Since, in gyrokinetics, $\omega / \Omega_{c} \sim \epsilon_{G K}$ is necessary for wave propagation, we are effectively considering a subsidiary electrostatic limit in which the wave in (4.12) does not propagate. When $\epsilon_{G K}^{2} v_{A, e} / c \ll$ $\sqrt{\beta}$, the same term is negligible, and we have

$$
\frac{\omega^{2}}{k_{\|}^{2} v_{A}^{2}}=\beta \frac{d_{e}^{2}}{\left(\rho_{e}^{2}+\lambda_{D}^{2}\right)\left[1+2 d_{e}^{2} k_{\perp}^{2}\right]},
$$

which, in the subsidiary $d_{e}^{2} k_{\perp}^{2} \rightarrow \infty$ limit, for $\lambda_{D} \gg \rho_{e}$ gives

$$
\omega^{2} \approx \frac{k_{\|}^{2}}{2 k_{\perp}^{2}} \omega_{p l}^{2}
$$

This result agrees with (4.4) and with the high-frequency solution of (3.7) of Helander \& Connor (2016). Our results suggest that, in a low-beta gyrokinetic theory, the displacement current can be neglected only if

$$
\epsilon_{G K}^{2} \frac{v_{A, e}}{c} \sim \epsilon_{G K}^{2} \frac{\lambda_{D}}{\rho_{e}} \ll \sqrt{\beta}
$$

${ }^{3}$ Notice that Helander \& Connor (2016) keep $\zeta \sim 1$. 


\section{Tearing instability}

We now consider an equilibrium magnetic field whose lines can reconnect. This is achieved if we add to the constant guide field a small component, $\boldsymbol{B}_{0} \rightarrow B_{0} \boldsymbol{e}_{z}+B_{y}(x) \boldsymbol{e}_{y}$, and $B_{y}$ changes sign for some $x=x_{r}$. The characteristic scale for the variation of the reconnecting field, $B_{y}$, is the shear length, $L_{s}$. At the location where reconnection occurs, $x \approx x_{r}$, for $x_{r}=0$, we have

$$
k_{\|} \approx k_{y} \frac{x}{L_{s}}
$$

Poisson's law and Ohm's law become, respectively

$$
\rho_{e}^{2} \frac{\partial^{2} \varphi}{\partial x^{2}}=-\frac{k_{y} v_{\text {the }}}{2 \omega} \frac{v_{\text {the }}}{c} \frac{x}{L_{s}} d_{e}^{2} \frac{\partial^{2} A_{\|}}{\partial x^{2}},
$$

and

$$
A_{\|}-\frac{k_{y} c}{\omega} \frac{x}{L_{s}} \varphi=\left(\mathrm{i} \frac{v}{\omega}+\frac{1}{2}-\frac{3}{4} \frac{k_{y}^{2} v_{\text {the }}^{2}}{\omega^{2}} \frac{x^{2}}{L_{s}^{2}}\right) d_{e}^{2} \frac{\partial^{2} A_{\|}}{\partial x^{2}},
$$

which can easily be cast in the form presented in Zocco \& Schekochihin (2011). Now, we have

$$
-\frac{x}{\delta}\left(A_{\|}-\frac{x}{\delta} \tilde{\varphi}\right) \sigma\left(\frac{x}{\delta}\right)=2 \rho_{e}^{2} \frac{\partial^{2} \tilde{\varphi}}{\partial x^{2}},
$$

and

$$
-\frac{x}{\delta} d_{e}^{2} \frac{\partial^{2} A_{\|}}{\partial x^{2}}=2 \rho_{e}^{2} \frac{\partial^{2} \tilde{\varphi}}{\partial x^{2}},
$$

where $\delta=L_{s} \omega /\left(k_{y} v_{\text {the }}\right), \tilde{\varphi}=\left(c / v_{\text {the }}\right) \varphi$, and

$$
\sigma\left(\frac{x}{\delta}\right)=\frac{1}{\mathrm{i} \frac{v}{\omega}+\frac{1}{2}-\frac{3}{4} \frac{x^{2}}{\delta^{2}}}
$$

Since we are always assuming $\rho_{e} \ll d_{e} \sim \delta$, we are effectively in a one-fluid limit, the ultralow beta discussed in Zocco \& Schekochihin (2011). We report on the collisionless case, the results apply to the collisional case in a straightforward manner. The analysis is known but we reproduce some key steps for the sake of clarity. One can introduce the function $\chi(\xi)=\xi A_{\|}^{\prime}(\xi)-A_{\|}(\xi)$, where $\xi=x / \delta_{\text {in }}$, and $\delta_{\text {in }}=\sqrt{4 \delta \rho_{e}^{2}}$, to obtain one equation for $\tilde{\chi}=-1+\chi / \chi_{0}$,

$$
\xi^{2} \frac{\mathrm{d}}{\mathrm{d} \xi}\left[\frac{1}{\xi^{2}}+\alpha^{2} G\right] \tilde{\chi}^{\prime}-\left(\xi^{2}+\lambda^{2}\right) \tilde{\chi}=\lambda^{2},
$$

where $\lambda^{2}=4 \delta \rho_{e} / d_{e}^{2}, \alpha=\sqrt{2 \rho / \delta}, G=\left(\delta^{2} / x^{2}\right)\left(\sigma^{-1}-2\right)$, the 'prime' is the derivative with respect to $\xi$, and $\chi_{0}$ is a constant of integration. The dispersion relation for the rescaled eigenvalue $\lambda^{2}$ is then

$$
\int_{0}^{\infty} \mathrm{d} \xi \frac{\tilde{\chi}^{\prime}}{\xi}=-\frac{\Delta^{\prime} \delta_{\text {in }}}{2}
$$

where $\Delta^{\prime}$ is the parameter that measures the discontinuity of the derivative of $A_{\|}^{\mathrm{MHD}}$ across the reconnection layer, and $A_{\|}^{\mathrm{MHD}}$ is the stable solution found in the ideal 
magnetohydrodynamics (MHD) region, $x \rightarrow \infty$ so that $E_{\|} \rightarrow 0$ (Furth, Killeen \& Rosenbluth 1963). As already pointed out in Zocco \& Schekochihin (2011), there is no need to solve (5.7) exactly to predict how the eigenvalue $\lambda^{2}$, hence the reconnection rate $\gamma$, scales with physical quantities. For $\rho_{e} \ll \delta$, from (5.7), we have $\tilde{\chi}^{\prime} / \xi \sim \lambda^{2}$. Since $\xi \sim 1$, then $\int_{0}^{\infty}\left(\tilde{\chi}^{\prime} / \xi\right) \sim \lambda^{2}$, which implies

$$
\frac{\gamma}{k_{y} v_{\text {the }}} \sim\left(\Delta^{\prime} d_{e}\right)^{2} \frac{d_{e}^{2}}{\rho_{e} L_{s}},
$$

where $\gamma=\operatorname{Im}[\omega]$. This is the equivalent of the collisionless result found for electronion plasmas by Drake \& Lee (1977), where the Alfvén speed is based on the electron mass. The collisional counterpart is recovered by replacing $d_{e} \rightarrow \sqrt{v d_{e}^{2} / \gamma}$, to obtain the traditional result of Furth et al. (1963) (but based on the electron Alfvén speed). When $\Delta^{\prime} \delta_{\text {in }} \gg 1$, the current is limited by the scale $\delta_{\text {in }}$, so that $\partial_{x}^{2} A_{\|} \sim A_{\|} / \delta_{\text {in }}^{2}$. Then, one replaces $\Delta^{\prime} \rightarrow \delta_{\text {in }}^{-1}$ in (5.8) to obtain $\lambda^{2} \sim 1$, which yields (Basu \& Coppi 1981)

$$
\frac{\gamma}{k_{y} v_{\text {the }}} \sim \frac{d_{e}^{2}}{\rho_{e} L_{s}},
$$

which gives the scaling $\gamma \sim\left(k_{y} v_{A, e}\right)^{2 / 3}\left(v d_{e}^{2} / L_{s}^{2}\right)^{1 / 3}$ of Coppi et al. (1976) in the collisional limit.

\section{Improved nonlinear model}

The inclusion of the Larmor scale in (2.5), instead of the Debye length, allowed us to avoid a covariant treatment. The use of the drift-kinetic model of Zocco \& Schekochihin (2011) helped us, but we did not exploit its full nonlinear potential yet. For this, fields amplitudes must be ordered more carefully. Equation (2.1), in fact, is nothing more than a drift-kinetic equation that one could have considered regardless of the results of Zocco \& Schekochihin (2011). If one wants to consider the nonlinear $\boldsymbol{E} \times \boldsymbol{B}$ frequency $\omega \sim\left(c / B_{0}\right) k^{2} \varphi$ and the streaming term, after using (2.5), we find that

$$
\frac{\delta n}{n_{0}} \sim \sqrt{\beta} \epsilon_{G K} .
$$

So, a nonlinear electromagnetic gyrokinetic theory that retains inertial effects must be developed to first order in a small Larmor radius expansion. This also implies that magnetic compressibility now must be retained to first order, since

$$
\frac{\delta B_{\|}}{B_{0}} \sim \beta \frac{e \varphi}{T_{0}} \sim \sqrt{\beta} \epsilon_{G K} \sim \frac{\delta n}{n_{0}} .
$$

Perpendicular magnetic fluctuations are ordered by balancing the electrostatic and the vector potential amplitudes of the gyrokinetic potential $\chi=\varphi-\left(v_{\|} / c\right) A_{\|}$, then

$$
\frac{\delta B_{\perp}}{B_{0}} \sim \frac{u_{\perp}}{v_{A, e}} \frac{1}{\sqrt{\beta}},
$$

where $u_{\perp} \sim c k_{\perp} \varphi / B_{0}$. In many relevant situations, the spatial variation of all quantities along the exact magnetic field is required, then $k_{\perp} \delta B_{\perp} \sim k_{\|} B_{0}$, which implies

$$
\frac{u_{\perp}}{v_{A, e}} \sim \sqrt{\beta} \epsilon_{G K} \rightarrow k_{\perp} \rho_{e} \sim \sqrt{\beta}
$$


and is naturally consistent with our fundamental ordering $k_{\perp} d_{e} \sim 1$. We therefore use

$$
\frac{\delta B_{\perp}}{B_{0}} \sim \epsilon_{G K},
$$

which is different from what the continuity equation (2.2) would have implied

$$
\frac{\delta B_{\perp}}{B_{0}} \sim \beta \epsilon_{G K} .
$$

Having completed the amplitude orderings, in order to obtain fluid-like equations, one can separate the first two moments of the perturbed distribution function, but considering first-order terms

$$
\begin{aligned}
h_{e^{\mp}}= & {\left[\frac{e^{\mp}\left(\varphi^{(0)}+\varphi^{(1)}\right)}{T_{0}}+\left(\frac{\delta n_{e^{\mp}}^{(0)}}{n_{0}}+\frac{\delta n_{e^{\mp}}^{(1)}}{n_{0}}\right)+2 \frac{v_{\|}\left(u_{\| e^{\mp}}^{(0)}+u_{\| e^{\mp}}^{(1)}\right)}{v_{\text {the }}^{2}}\right] F_{0} } \\
& +g^{(0)}+g^{(1)}+O\left(k_{\perp}^{4} \rho_{e}^{4}\right),
\end{aligned}
$$

where $\int \mathrm{d}^{3} \boldsymbol{v} g=\int \mathrm{d}^{3} \boldsymbol{v} v_{\|} g \equiv 0$ to all orders in $k_{\perp}^{2} \rho_{e}^{2} \sim \beta \ll 1$. When magnetic compressibility is retained, the gyrokinetic potential on the right-hand side of (2.1) becomes

$$
\varphi-\frac{v_{\|}}{c} A_{\|} \rightarrow \varphi-\frac{v_{\|}}{c} A_{\|}+\frac{T_{0}}{e} \hat{v}_{\perp}^{2} \frac{\delta B_{\|}}{B_{0}} \equiv \chi,
$$

where

$$
\frac{\delta B_{\|}}{B_{0}}=-\beta \sum_{\mp} \int \mathrm{d}^{3} v \hat{v}_{\perp}^{2} h_{e^{\mp}} .
$$

We notice that, due to its parity in velocity space, the new $\delta B_{\|}$term enters in the equation for density fluctuations. Let us evaluate the density moment of (2.1) after using (6.8), and subtract the two results obtained, one for each species. To leading order we have

$$
\hat{\boldsymbol{b}} \cdot \nabla d_{e}^{2} \nabla^{2} A_{\|}^{(0)}=0 .
$$

To next order, (2.4) gives

$$
\left(\frac{\delta n_{e^{+}}^{(1)}}{n_{0}}-\frac{\delta n_{e^{-}}^{(1)}}{n_{0}}\right)=-\frac{\rho_{e}^{2} \nabla^{2}}{2} \frac{e \varphi^{(0)}}{T_{0}}-\frac{\rho_{e}^{2} \nabla^{2}}{4}\left(\frac{\delta T_{\perp e^{+}}^{(0)}}{T_{0}}-\frac{\delta T_{\perp e^{-}}^{(0)}}{T_{0}}\right),
$$

which now proves our previous statement on the validity of (2.5), since here

$$
\frac{\delta T_{\perp e^{\mp}}}{T_{0}}=\frac{1}{n_{0}} \int \mathrm{d}^{3} \boldsymbol{v} \hat{v}_{\perp}^{2} g_{e^{\mp}}
$$

are the perpendicular temperature fluctuations. As usual, they drive parallel magnetic compressional perturbations

$$
\frac{\delta B_{\|}^{(1)}}{B_{0}}=-\frac{\beta_{e}}{2}\left(\frac{\delta n_{e^{+}}^{(0)}}{n_{0}}+\frac{\delta n_{e^{-}}^{(0)}}{n_{0}}+\frac{\delta T_{\perp, e^{-}}^{(0)}}{T_{0}}+\frac{\delta T_{\perp, e^{+}}^{(0)}}{T_{0}}\right),
$$

which have to be taken into account in the first-order continuity equation. The electrostatic potential is determined by the difference of the first-order density 
fluctuations of the two species. We then subtract the two first-order continuity equations, and obtain the generalised vorticity equation

$$
\begin{aligned}
\frac{\mathrm{d}}{\mathrm{d} t}[ & \left.\rho_{e}^{2} \nabla^{2} \frac{e \varphi^{(0)}}{T_{0}}-\frac{\rho_{e}^{2} \nabla^{2}}{4}\left(\frac{\delta T_{\perp e^{-}}^{(0)}}{T_{0}}-\frac{\delta T_{\perp e^{+}}^{(0)}}{T_{0}}\right)-\beta_{e}\left(\frac{\delta T_{\perp e^{-}}^{(0)}}{T_{0}}+\frac{\delta T_{\perp e^{+}}^{(0)}}{T_{0}}\right)\right] \\
= & -\hat{\boldsymbol{b}} \cdot \nabla\left[\frac{e}{m c} d_{e}^{2} \nabla^{2} A_{\|}^{(1)}+\beta_{e}\left(u_{\| e^{-}}^{(0)}+u_{\| e^{+}}^{(0)}\right)\right]+\frac{1}{B_{0}}\left\{A_{\|}^{(1)}+\frac{\rho_{e}^{2} \nabla^{2}}{4} A_{\|}^{(0)}, \frac{e}{m c} d_{e}^{2} \nabla^{2} A_{\|}^{(0)}\right\} \\
& -\frac{c T_{0}}{B_{0} e}\left\{\frac{\rho_{e}^{2} \nabla^{2}}{4} \frac{e \varphi^{(0)}}{T_{0}}+\frac{\delta B_{\|}^{(1)}}{B_{0}}, \frac{\delta T_{\perp e^{-}}^{(0)}}{T_{0}}-\frac{\delta T_{\perp e^{+}}^{(0)}}{T_{0}}\right\} \\
& +\frac{v_{\text {the }}}{B_{0}}\left\{\frac{\rho_{e}^{2} \nabla^{2}}{4} A_{\|}^{(0)}, \frac{1}{n_{0}} \int \mathrm{d}^{3} v \hat{v}_{\|} \hat{v}_{\perp}^{2}\left(g_{e^{-}}^{(0)}-g_{e^{+}}^{(0)}\right)\right\},
\end{aligned}
$$

where the terms multiplying an explicit $\beta_{e}$ come from (6.13). We now evaluate all the terms of the right-hand side. To zeroth order, (2.8) is valid, and we have

$$
\begin{aligned}
\frac{\mathrm{d}}{\mathrm{d} t}\left(u_{\| e^{+}}^{(0)}+u_{e^{-}}^{(0)}\right)= & -\frac{v_{\text {the }}^{2}}{2} \hat{\boldsymbol{b}} \cdot \nabla\left(\frac{\delta n_{e^{+}}^{(0)}}{n_{0}}+\frac{\delta n_{e^{-}}^{(0)}}{n_{0}}+\frac{\delta T_{\perp, e^{-}}^{(0)}}{T_{0}}+\frac{\delta T_{\perp, e^{+}}^{(0)}}{T_{0}}\right) \\
& +\frac{v_{\text {the }}^{2}}{L_{n}}\left(1+\eta_{e}\right) \frac{\partial_{y} A_{\|}^{(0)}}{B_{0}},
\end{aligned}
$$

where we see that diamagnetic effects do not cancel. The sum $\delta n_{e^{+}}^{(0)}+\delta n_{e^{-}}^{(0)}$ can be evaluated by using the zeroth-order continuity equations

$$
\frac{\mathrm{d}}{\mathrm{d} t}\left(\frac{\delta n_{e^{+}}^{(0)}}{n_{0}}+\frac{\delta n_{e^{-}}^{(0)}}{n_{0}}\right)=-\hat{\boldsymbol{b}} \cdot \nabla\left(u_{\| e^{+}}^{(0)}+u_{e^{-}}^{(0)}\right)-\frac{v_{\text {the }}}{L_{n}} \rho_{e} \partial_{y} \frac{e \varphi^{(0)}}{T_{0}} .
$$

To first order, only the difference $u_{\| e^{-}}^{(1)}-u_{\| e^{+}}^{(1)}=(e / m c) d_{e}^{2} \nabla^{2} A_{\|}^{(1)}$ enters the vorticity equation, thus we obtain

$$
\begin{aligned}
\frac{\mathrm{d}}{\mathrm{d} t} & \left(A_{\|}^{(1)}-\frac{d_{e}^{2} \nabla^{2}}{2} A_{\|}^{(1)}+\frac{\rho_{e}^{2} \nabla^{2}}{4} A_{\|}^{(0)}\right) \\
& =-c \hat{\boldsymbol{b}} \cdot \nabla \varphi^{(1)}+\frac{c T_{0}}{2 e} \hat{\boldsymbol{b}} \cdot \nabla\left[\frac{\delta n_{e^{-}}^{(0)}}{n_{0}}-\frac{\delta n_{e^{+}}^{(0)}}{n_{0}}+\frac{\delta T_{\| e^{-}}^{(1)}}{T_{0}}-\frac{\delta T_{\| e^{+}}^{(1)}}{T_{0}}\right] \\
& +\frac{c}{B_{0}}\left\{\varphi^{(1)}+\frac{\rho_{e}^{2} \nabla^{2}}{4} \varphi^{(0)}+\frac{T_{0}}{e} \frac{\delta B_{\|}^{(1)}}{B_{0}}, \frac{d_{e}^{2} \nabla^{2}}{2} A_{\|}^{(0)}\right\}-\frac{c T_{0}}{e B_{0}}\left\{A_{\|}^{(1)}, \frac{\delta T_{\| e^{-}}^{(0)}}{T_{0}}-\frac{\delta T_{\| e^{+}}^{(0)}}{T_{0}}\right\} \\
& -\frac{c T_{0}}{e B_{0}}\left\{\frac{\rho_{e}^{2} \nabla^{2}}{4} A_{\|}^{(0)}, \frac{1}{n_{0}} \int \mathrm{d}^{2} \boldsymbol{v} \hat{v}_{\|}^{2} \hat{v}_{\perp}^{2}\left(g_{e^{-}}^{(0)}-g_{e^{+}}^{(0)}\right)\right\} \\
& +\frac{c}{B_{0}}\left\{\frac{\rho_{e}^{2} \nabla^{2}}{4} \varphi^{(0)}+\frac{T_{0}}{e} \frac{\delta B_{\|}^{(1)}}{B_{0}}, \frac{m c}{2 e} \frac{1}{n_{0}} \int \mathrm{d}^{3} \boldsymbol{v} v_{\|} \hat{v}_{\perp}^{2}\left(g_{e^{-}}^{(0)}-g_{e^{+}}^{(0)}\right)\right\} \\
& -\frac{c m}{2 e n_{0}} \int \mathrm{d}^{3} \boldsymbol{v} v_{\|} \sum_{s}\left(\frac{\partial h_{s}}{\partial t}\right)_{\mathrm{coll}} \cdot
\end{aligned}
$$


An equation for $g^{(0)}$ is derived by subtracting (2.2) and (2.8) from the gyrokinetic equation, and using (6.7). The result is

$$
\begin{aligned}
& \frac{\mathrm{d}}{\mathrm{d} t} g_{e^{\mp}}^{(0)}+v_{\|} \hat{\boldsymbol{b}} \cdot \nabla\left(g_{e^{\mp}}^{(0)}-\frac{\delta T_{\| e^{\mp}}^{(0)}}{T_{0}} F_{0}\right)-C\left[g_{e^{\mp}}^{(0)}\right] \\
& \quad=\left(1-2 \frac{v_{\|}^{2}}{v_{\text {the }}^{2}}\right) \hat{\boldsymbol{b}} \cdot \nabla u_{\| e^{\mp}}^{(0)} F_{0}-\frac{c T_{0}}{e B_{0}} \frac{\partial_{y}}{L_{T}}\left[\left(\hat{v}^{2}-\frac{3}{2}\right) \frac{e \varphi^{(0)}}{T_{0}}-\left(\hat{v}^{2}-\frac{5}{2}\right) \frac{v_{\|}}{c} \frac{e A_{\|}^{(0)}}{T_{0}}\right] F_{0},
\end{aligned}
$$

where

$$
C\left[g_{e^{\mp}}^{(0)}\right]=\left(\frac{\partial h_{e^{\mp}}^{(0)}}{\partial t}\right)_{\text {coll }}-2 \frac{v_{\|} F_{0}}{v_{\text {the }}^{2} n_{0}} \int \mathrm{d}^{3} \boldsymbol{v}\left(\frac{\partial h_{e^{\mp}}^{(0)}}{\partial t}\right)_{\text {coll }},
$$

and $\hat{v}^{2}=\hat{v}_{\|}^{2}+\hat{v}_{\perp}^{2}$. Equation (6.18) is the extension of the kinetic reduced electron heating model equation (Zocco \& Schekochihin 2011) to the case of finite density and temperature gradients (Zocco et al. 2015; Loureiro et al. 2016). For the evolution of the fields, again, the important quantities are the difference and the sum the electron and positron equations. From the difference we obtain

$$
\frac{\mathrm{d}}{\mathrm{d} t}\left(g_{e^{-}}^{(0)}-g_{e^{+}}^{(0)}\right)+v_{\|} \hat{\boldsymbol{b}} \cdot \nabla\left[g_{e^{-}}^{(0)}-g_{e^{+}}^{(0)}-\left(\frac{\delta T_{\| e^{-}}^{(0)}}{T_{0}}-\frac{\delta T_{\| e^{+}}^{(0)}}{T_{0}}\right) F_{0}\right]=C\left[g_{e^{\mp}}^{(0)}-g_{e^{+}}^{(0)}\right]
$$

where we used the fact that $\hat{\boldsymbol{b}} \cdot \nabla \nabla^{2} A_{\|}^{(0)}=0$, and the diamagnetic cancellation. On the other hand, the sum gives

$$
\begin{aligned}
\frac{\mathrm{d}}{\mathrm{d} t}\left(g_{e^{-}}^{(0)}+g_{e^{+}}^{(0)}\right)+v_{\|} \hat{\boldsymbol{b}} \cdot \nabla\left[g_{e^{-}}^{(0)}+g_{e^{+}}^{(0)}-\left(\frac{\delta T_{\| e^{-}}^{(0)}}{T_{0}}+\frac{\delta T_{\| e^{+}}^{(0)}}{T_{0}}\right) F_{0}\right] \\
=C\left[g_{e^{+}}^{(0)}+g_{e^{-}}^{(0)}\right]+\left(1-2 \frac{v_{\|}^{2}}{v_{\text {the }}^{2}}\right) \hat{\boldsymbol{b}} \cdot \nabla\left(u_{\| e^{-}}^{(0)}+u_{\| e^{+}}^{(0)}\right) F_{0} \\
-2 \frac{c T_{0}}{e B_{0}} \frac{\partial_{y}}{L_{T}}\left[\left(\hat{v}^{2}-\frac{3}{2}\right) \frac{e \varphi^{(0)}}{T_{0}}-\left(\hat{v}^{2}-\frac{5}{2}\right) \frac{v_{\|}}{c} \frac{e A_{\|}^{(0)}}{T_{0}}\right] F_{0}
\end{aligned}
$$

and the diamagnetic contribution does not cancel. It remains to specify the collisional operator. We notice that, for sufficiently large collisionality, temperature fluctuations must become isotropic, that is $\delta T_{\perp}=\delta T_{\|}$. This is achieved with the simple collision operator model

$$
\left(\frac{\partial h_{e^{\mp}}}{\partial t}\right)_{\text {coll }}=v\left\{\frac{1}{2} \frac{\partial}{\partial \hat{v}_{\|}}\left(\frac{\partial}{\partial \hat{v}_{\|}}+\hat{v}_{\|}\right) h_{e^{\mp}}+2 \frac{v_{\|} u_{\|, e^{\mp}}}{v_{\text {the }}^{2}}+\left(1-2 \hat{v}_{\|}^{2}\right) \frac{\delta T_{\perp, e \mp}}{T_{0}} F_{0}\right\} .
$$


Finally, with the equation for $g_{e^{\mp}}^{(1)}$, we can close our system. This is obtained from

$$
\begin{aligned}
\frac{\partial h_{\mp}^{(1)}}{\partial t} & +v_{\|} \frac{\partial h_{\mp}^{(1)}}{\partial z}+\frac{c}{B_{0}}\left\{\chi^{(0)}, h_{\mp}^{(1)}\right\}+\frac{c}{B_{0}}\left\{\chi^{(1)}+\hat{v}_{\perp}^{2} \frac{\rho_{e}^{2} \nabla^{2}}{4} \chi^{(0)}, h_{\mp}^{(0)}\right\} \\
= & \frac{e^{\mp} F_{0}}{T_{0}} \frac{\partial}{\partial t}\left(\chi^{(1)}+\hat{v}_{\perp}^{2} \frac{\rho_{e}^{2} \nabla^{2}}{4} \chi^{(0)}\right)-\frac{c n_{0}}{B_{0} L_{n}} \partial_{y}\left(\chi^{(1)}+\hat{v}_{\perp}^{2} \frac{\rho_{e}^{2} \nabla^{2}}{4} \chi^{(0)}\right) \\
& \times\left[1+\eta_{e^{\mp}}\left(\hat{v}^{2}-\frac{3}{2}\right)\right] F_{0}+\left(\frac{\partial h_{\mp}^{(1)}}{\partial t}\right)_{\text {coll. }},
\end{aligned}
$$

where the equations for all the fields have already been derived. We leave this expression implicit, and proceed considering the limiting case of collisionless isothermal species. This is the limit in which the KREHM equations (Zocco \& Schekochihin 2011) reduce to the Schep Pegoraro and Kuvshinov two-fluid model (Schep et al. 1994). However, now we expect a new result. Indeed, even if our quasi-neutrality equation, (6.11), looks similar to a long-wavelength electrostatic ion response

$$
\delta n_{e^{-}}^{(1)}-\delta n_{e^{+}}^{(1)} \propto \rho_{e}^{2} \nabla^{2} \varphi \rightarrow \delta n_{e}^{(0)}=\delta n_{i}^{(0)} \propto \nabla^{2} \rho_{i}^{2} \varphi,
$$

its physical content is rather different. Indeed, the equation for the electrostatic potential (which, for pair plasmas, must come from the first-order quasi-neutrality condition, while for electron-ion plasmas comes from the zeroth-order quasi-neutrality condition), in both cases, has the form of a vorticity equation (6.14). However, finite Larmor radius effects introduce a magnetic compressional dynamics that will manifest and generate a qualitatively different set of equations from the equivalent electron-ion ones. Let us derive this new set of nonlinear equations for collisionless magnetic reconnection and turbulence in magnetised isothermal pair plasmas. We want to stress that the isothermal collisionless limit is a crude approximation and its validity should always be tested against the solution of the reduced kinetic problem, (6.23), as it has been done for electron-ion plasmas by Loureiro et al. (2013).

We introduce the field-line-following coordinate, $l$, and integrate (6.10) along the perturbed field lines. Since, for $l \rightarrow \mp \infty$, the solution must decay to zero, we set to zero the resulting constant of integration. The electric field, therefore, to zeroth order satisfies the ideal MHD equation

$$
\frac{\partial}{\partial t} A_{\|}^{(0)}+c \hat{\boldsymbol{b}} \cdot \nabla \varphi^{(0)}=0
$$

with

$$
\nabla^{2} A_{\|}^{(0)}=0
$$

We are considering the isothermal response, thus, $g_{e^{\mp}} \equiv 0$. By inspecting (6.18) and (6.23), we observe that they are homogeneous, and therefore consistent with these solutions, only if $L_{n} \rightarrow \infty$. We consider this limit.

Then, the generalised Ohm's law reduces to

$$
\frac{\mathrm{d}}{\mathrm{d} t}\left(A_{\|}^{(1)}-\frac{d_{e}^{2} \nabla^{2}}{2} A_{\|}^{(1)}\right)=-c \hat{\boldsymbol{b}} \cdot \nabla \varphi^{(1)}+\frac{C T_{0}}{2 e} \hat{\boldsymbol{b}} \cdot \nabla \frac{\rho_{e}^{2} \nabla^{2}}{2} \varphi^{(0)},
$$


while the vorticity equation gives

$$
\frac{\mathrm{d}}{\mathrm{d} t} \rho_{e}^{2} \nabla^{2} \frac{e \varphi^{(0)}}{T_{0}}=-\hat{\boldsymbol{b}} \cdot \nabla\left[\frac{e}{m c} d_{e}^{2} \nabla^{2} A_{\|}^{(1)}+\beta_{e} V_{\|}^{(0)}\right] .
$$

Due to finite magnetic compressibility, this is coupled to the equations for the total density $2 \delta n^{(0)}=\delta n_{e^{-}}^{(0)}+\delta n_{e^{+}}^{(0)}$ and the velocity $V_{\|}^{(0)}=u_{\| e^{-}}^{(0)}+u_{\| e^{+}}^{(0)}$

$$
\begin{gathered}
\frac{\mathrm{d}}{\mathrm{d} t} V_{\|}^{(0)}=-v_{\mathrm{the}}^{2} \hat{\boldsymbol{b}} \cdot \nabla \frac{\delta n^{(0)}}{n_{0}}, \\
2 \frac{\mathrm{d}}{\mathrm{d} t} \frac{\delta n^{(0)}}{n_{0}}=-\hat{\boldsymbol{b}} \cdot \nabla V_{\|}^{(0)} .
\end{gathered}
$$

We found a set of six equations for the six unknowns $A_{\|}^{(0)}, \varphi^{(0)}, A_{\|}^{(1)}, \varphi^{(1)}, V_{\|}^{(0)}$ and $\delta n^{(0)}$. We see that compressible effects enter the vorticity equation (6.28) through $\beta_{e}$ because quasi-neutrality is valid to first order in the small Larmor radius expansion. The new term, proportional to $V_{\|}=u_{\| e^{-}}^{(0)}+u_{\| e^{+}}^{(0)}$, couples the electromagnetic system of equations for $\varphi^{(1)}$ and $A_{\|}^{(1)}$ to the fluid equations (6.29) and (6.30). It is easy to show that these equations support the compressional sound wave $\omega=v_{\text {the }} k_{\|} / \sqrt{2}$, which is linearly strongly damped in the electrostatic limit (Helander \& Connor 2016; Mishchenko et al. 2017). In the low-frequency limit $\omega \ll k_{y} v_{\text {the }}\left(\partial_{x} A_{\|}^{(0)} / B_{0}\right) \sim k_{z} v_{\text {the }}$, such damping is negligible. Thus, (6.25)-(6.30) are valid for sub-Alfvénic instabilities, $\omega \sim k_{y} v_{A, e} \lambda$, with $\lambda \ll \sqrt{\beta_{e}} \ll 1$, provided nonlinear collisionless heating is negligible $\left(g_{e^{\mp}} \equiv 0\right)$. This is definitely true for the reconnecting instabilities presented in $\S 5$.

\section{Conclusion}

We presented a simple study of non-relativistic electron-positron plasmas in a magnetised sheared slab. The two species were described by using the drift-kinetic model. Linear results are presented for nearly electrostatic perturbations, $\delta B_{\perp} / B_{0} \sim$ $\beta \epsilon_{G K} \ll e \varphi / T_{0} \sim \epsilon_{G K}$, where $\epsilon_{G K}=k_{\|} / k_{\perp}$ is the small expansion parameter of gyrokinetic theory, $k_{\|}$and $k_{\perp}$ are the wave vectors for perturbations parallel and perpendicular to the equilibrium magnetic field, $B_{0}$, and $\beta$ is the ratio of kinetic to magnetic plasma pressure. We emphasised the role of the electron Alfvén wave. This is supported by the system and connects, in the electrostatic limit, to high-frequency K-modes. Current driven instabilities are described by a formalism equivalent to that of an electron-ion plasma, but with the Alfvén speed based on the electron mass. A nonlinear electromagnetic model is also presented. Here, nonlinear frequencies associated with electrostatic fluctuations are allowed to compete with the characteristic frequencies of wave-particle resonant interaction. The resulting hybrid fluid-kinetic model is an extension of the KREHM of Zocco \& Schekochihin (2011), where finite Larmor radius effects are retained. The specific case of isothermal species is derived in detail, and its limitations are discussed. This constitutes a new set of equations for nonlinear electromagnetic phenomena in magnetised pair plasmas. The results are, at present, highly speculative. The first magnetically confined pair plasmas will definitely be dominated by electrostatic effects, because of their extremely low density. Our nonlinear electromagnetic model could perhaps be a useful tool to design high density magnetically confined electron-positron plasmas. 


\section{Acknowledgements}

I am grateful to M. Barnes, J. Connor, P. Helander, N. Loureiro, A. Mishchenko, A. Schekochihin and T. Sunn Pedersen for valuable discussions that helped me understand many aspects of this work. Part of this work was presented at the first conference 'Frontiers in Plasma Physics', held at the Abbey of the Most Holy Trinity of Spineto (Sarteano, Italy) under the auspices of the Journal of Plasma Physics (Cambridge University Press).

\section{REFERENCES}

BASU, B. \& COPPI, B. 1981 Theory of $m=l$ modes in collisionless plasmas. Phys. Fluids 24 (3), $465-471$.

Bessho, N. \& Bhattacharjee, A. 2005 Collisionless reconnection in an electron-positron plasma. Phys. Rev. Lett. 95 (24), 245001-245004.

BraginskiI, S. I. 1965 Transport processes in a plasma. In Reviews of Plasma Physics (ed. M. A. Leontovich), vol. 1, pp. 205-311. Consultants Bureau.

Chacón, L., Simakov, A. N., Lukin, V. S.\& Zocco, A. 2008 Fast reconnection in nonrelativistic 2d electron-positron plasmas. Phys. Rev. Lett. 101 (2), 025003.

Chacón, L., Simakov, A. N. \& Zocco, A. 2007 Steady-state properties of driven magnetic reconnection in 2d electron mhd. Phys. Rev. Lett. 99, 235001.

Coppi, B., Galvão, R. M. O., Pellat, R., Rosenbluth, M. N. \& Rutherford, P. H. 1976 Fiz. Plazmy 6, 691; Sov. J. Plasma Phys. 11, 226 (1975).

Daughton, W. \& Karimabadi, H. 2007 Collisionless magnetic reconnection in large-scale electronpositron plasmas. Phys. Plasmas 14 (7), 72303-72310.

Drake, J. F. \& LeE, Y. C. 1977 Kinetic theory of tearing instabilities. Phys. Fluids 20 (8), 1341-1353.

Fried, B. D., Hendrick, C. L. \& MCCune, J. 1968 Two-pole approximation for the plasma dispersion function. Phys. Fluids 11, 249-252.

FriemAn, E. A. \& CHEN, L. 1982 Nonlinear gyrokinetic equations for low-frequency electromagnetic waves in general plasma equilibria. Phys. Fluids 25 (3), 502-508.

Furth, H. P., Killeen, J. \& Rosenbluth, M. N. 1963 Finite-resistivity instabilities of a sheet pinch. Phys. Fluids 6, 1169.

Guo, F., Li, H., Daughton, W., Li, X. \& LiU, Y.-H. 2016 Particle acceleration during magnetic reconnection in a low-beta pair plasma. Phys. Plasmas 23 (5), 055708.

Helander, P. 2014 Microstability of magnetically confined electron-positron plasmas. Phys. Rev. Lett. 113, 135003.

Helander, P. 2017 Available energy and ground states of collisionless plasmas. J. Plasma Phys. 83 (4), 715830401.

Helander, P. \& Connor, J. W. 2016 Gyrokinetic stability theory of electron-positron plasmas. J. Plasma Phys. 82 (3), 905820301.

Lenard, A. \& Bernstein, I. B. 1958 Plasma oscillations with diffusion in velocity space. Phys. Rev. 112 (5), 1456.

Loureiro, N. F., Dorland, W., Fazendeiro, L., Kanekar, A., Mallet, A., Vilelas, M. S. \& ZocCO, A. 2016 Viriato: a Fourier-Hermite spectral code for strongly magnetized fluid kinetic plasma dynamics. Comput. Phys. Commun. 206, 45-63.

Loureiro, N. F., Schekochinin, A. A. \& Zocco, A. 2013 Fast collisionless reconnection and electron heating in strongly magnetized plasmas. Phys. Rev. Lett. 111, 025002.

Makwana, K. D., Zhdankin, V., Li, H., Daughton, W. \& Cattaneo, F. 2015 Energy dynamics and current sheet structure in fluid and kinetic simulations of decaying magnetohydrodynamic turbulence. Phys. Plasmas 22 (4), 042902.

Mishchenko, A., Zocco, A., Helander, P. \& Könies, A. 2017 J. Plasma Phys. (submitted). 
Ottaviani, M. \& Porcelli, F. 1995 Fast nonlinear magnetic reconnection. Phys. Plasmas 2 (11), $4104-4117$.

Pedersen, T. S., Danielson, J. R., Hugenschmidt, C., Marx, G., Sarasola, X., Schauer, F., Schweikhard, L., Surko, C. M. \& Winkler, E. 2012 Plans for the creation and studies of electron-positron plasmas in a stellarator. New J. Phys. 14 (3), 035010.

Saitoh, H., Pedersen, T. S., Hergenhahn, U., Stenson, E. V., Paschkowski, N. \& Hugenschmidt, C. 2014 Recent status of a positron-electron experiment (apex). J. Phys. Conf. Ser. 505 (1), 012045.

Schekochihin, A. A., Parker, J. T., Highcock, E. G., Dellar, P. J., Dorland, W. \& Hammett, G. W. 2016 Phase mixing versus nonlinear advection in drift-kinetic plasma turbulence. J. Plasma Phys. 82 (2), 905820212.

Schep, T. J., Pegoraro, F. \& Kuvshinov, B. N. 1994 Generalized two-fluid theory of nonlinear magnetic structures. Phys. Plasmas 1 (9), 2843-2852.

Stenson, E. V., Horn-Stanja, J., Stoneking, M. R. \& Pedersen, T. S. 2017 Debye length and plasma skin depth: two length scales of interest in the creation and diagnosis of laboratory pair plasmas. J. Plasma Phys. 83 (1), 595830106.

Zocco, A. 2015 Linear collisionless Landau damping in Hilbert space. J. Plasma Phys. 81 (4), 7.

Zocco, A., Chacón, L. \& Simakov, A. 2008 Electron inertia effects in 2d driven reconnection in electron mhd. In Theory of Fusion Plasmas, AIP Conference Proceedings, vol. 1069, pp. 349-354. AIP.

Zocco, A., Chacón, L. \& Simakov, A. N. 2009 Current sheet bifurcation and collapse in electron magnetohydrodynamics. Phys. Plasmas 16 (11), 110703.

Zocco, A., Loureiro, N. F., Dickinson, D., Numata, R. \& Roach, C. M. 2015 Kinetic microtearing modes and reconnecting modes in strongly magnetised slab plasmas. Plasma Phys. Control. Fusion 57 (6), 065008.

Zocco, A. \& Schekochinin, A. A. 2011 Reduced fluid-kinetic equations for low-frequency dynamics, magnetic reconnection, and electron heating in low-beta plasmas. Phys. Plasmas $\mathbf{1 8}$ (10), 102309. 